\title{
May 2015 Imaging Case of the Month
}

\author{
Michael B. Gotway, MD \\ Department of Radiology \\ Mayo Clinic Arizona \\ Scottsdale, AZ
}

Clinical History: A 66 year-old woman presented with a history of hypothyroidism on replacement therapy, and a past medical history of pancreatitis, presented to her gastroenterologist with complaints of abdominal pain and loose stools. The episodes of pancreatitis began over a decade earlier with epigastric pain that was ultimately attributed to cholecystitis, for which endoscopic retrograde cholangiopancreatography (ERCP) was performed; this procedure precipitated her first episode of pancreatitis. During the ERCP procedure, her common bile duct was noted to be narrowed and several stones were removed, with placement of a stent, after which her epigastric pain resolved. A second stent placement procedure was required for recurrent epigastric pain approximately three weeks later, with good result.

Nearly a decade later, the patient presented with loose stools and fecal urgency associated with abdominal pain. Upper endoscopy showed mild gastric erosions (the patient was taking non-steroidal anti-inflammatory agents for intermittent back pain) and colonoscopy showed mild, non-specific colitis. The paint was diagnosed with pancreatic insufficiency and enzyme replacement therapy was begun, with symptomatic improvement.

During the course of her gastrointestinal consult, a frontal chest radiograph (Figure 1) was performed.

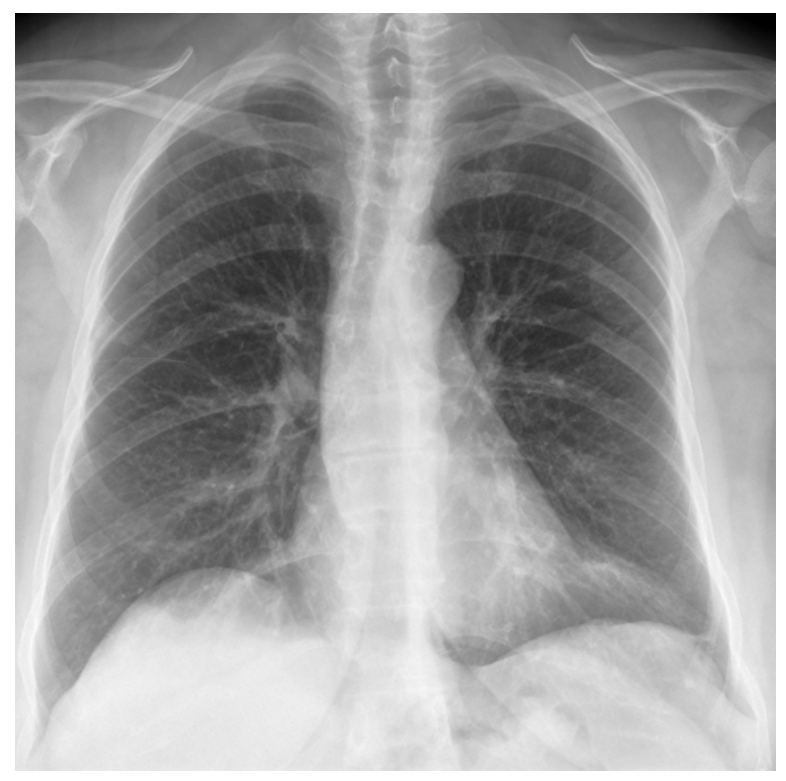

Figure 1. Frontal chest radiograph. 
Which of the following statements regarding the chest radiograph is most accurate?

1. The frontal chest radiograph shows a poorly defined opacity in the extreme right base

2. The frontal chest radiograph shows abnormal mediastinal contours

3. The frontal chest radiograph shows basal predominant fibrotic abnormalities

4. The frontal chest radiograph shows large lung volumes with a cystic appearance

5. The frontal chest radiograph shows no abnormal findings 


\section{Correct! \\ 1. The frontal chest radiograph shows a poorly defined opacity in the extreme right base}

The frontal chest radiograph shows faintly seen, poorly defined opacity in the inferior right thorax. This abnormality is difficult to appreciate, but note the relatively increased opacity seen "through" the right diaphragm, obscuring the vessels that should normally be visualized in this region. For example, compare the right base to the left base- note how branching vessels can be "seen through" the left diaphragm, but not nearly as well on the right.

As part of the evaluation of her abdominal complaints, the patient underwent enhanced abdominal CT. The lung bases and upper abdomen portions of this study are shown in Figure 2.

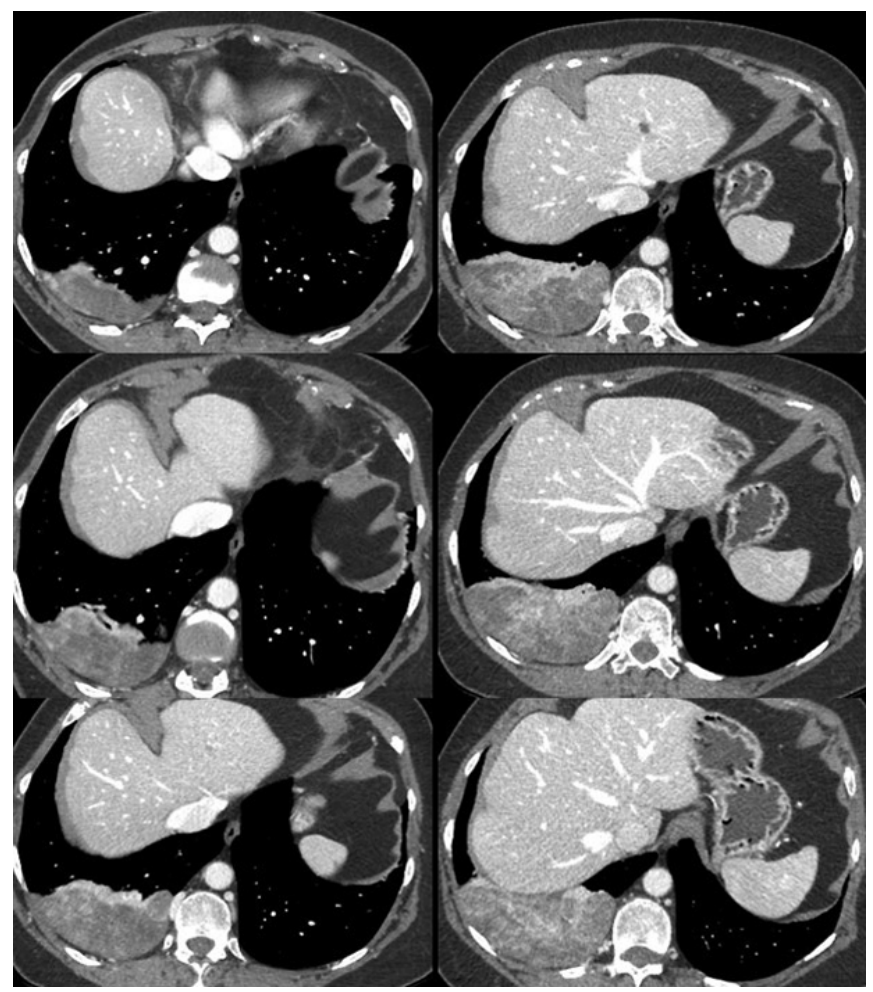

Figure 2. Axial abdominal CT images obtained during the evaluation of suspected pancreatic insufficiency.

Regarding this examination, which of the following is correct?

1. The abdominal CT shows a destructive right-sided chest wall mass

2. The abdominal CT shows a large right pleural effusion

3. The abdominal CT shows a posterior mediastinal mass

4. The abdominal CT shows a vascularized right base mass

5. The abdominal CT shows intrathoracic herniation of right-sided posterior subdiaphragmatic structures 


\section{Correct!}

\section{The abdominal CT shows a vascularized right base mass}

The abdominal CT shows a mass with broad contact with the right posterior chest wall, clearly containing some enhancing septae-like vascularity. The lesion is complex and vascularized and therefore does not represent simple right pleural liquid. While the mass does contact a significant portion of the right chest wall, there is no evidence of rib destruction or chest wall invasion. The mass does contact a portion of the posterior mediastinum (along the right aspect of the thoracic vertebral bodies), but the majority of the mass is centered more directly posteriorly, not within the typical boundaries of the posterior mediastinum. The mass is in a position within the thorax that is often affected by diaphragmatic defects that allow intrathoracic herniation of subdiaphragmatic contents. However, the mass is an abnormal finding and does not represent intrathoracic herniation of an otherwise normal abdominal or retroperitoneal organ, and no diaphragmatic defect is evident.

Clinical Course: Following discovery of the mass at abdominal CT scanning, the patient was questioned regarding possible symptoms that could be related to the mass, but she denied night sweats, cough, dyspnea, weight loss, palpitations, hemoptysis, and chest pain. At abdominal CT, a small area of heterogeneous pancreatic enhancement was seen, for which MR (Figure 3) evaluation was suggested.

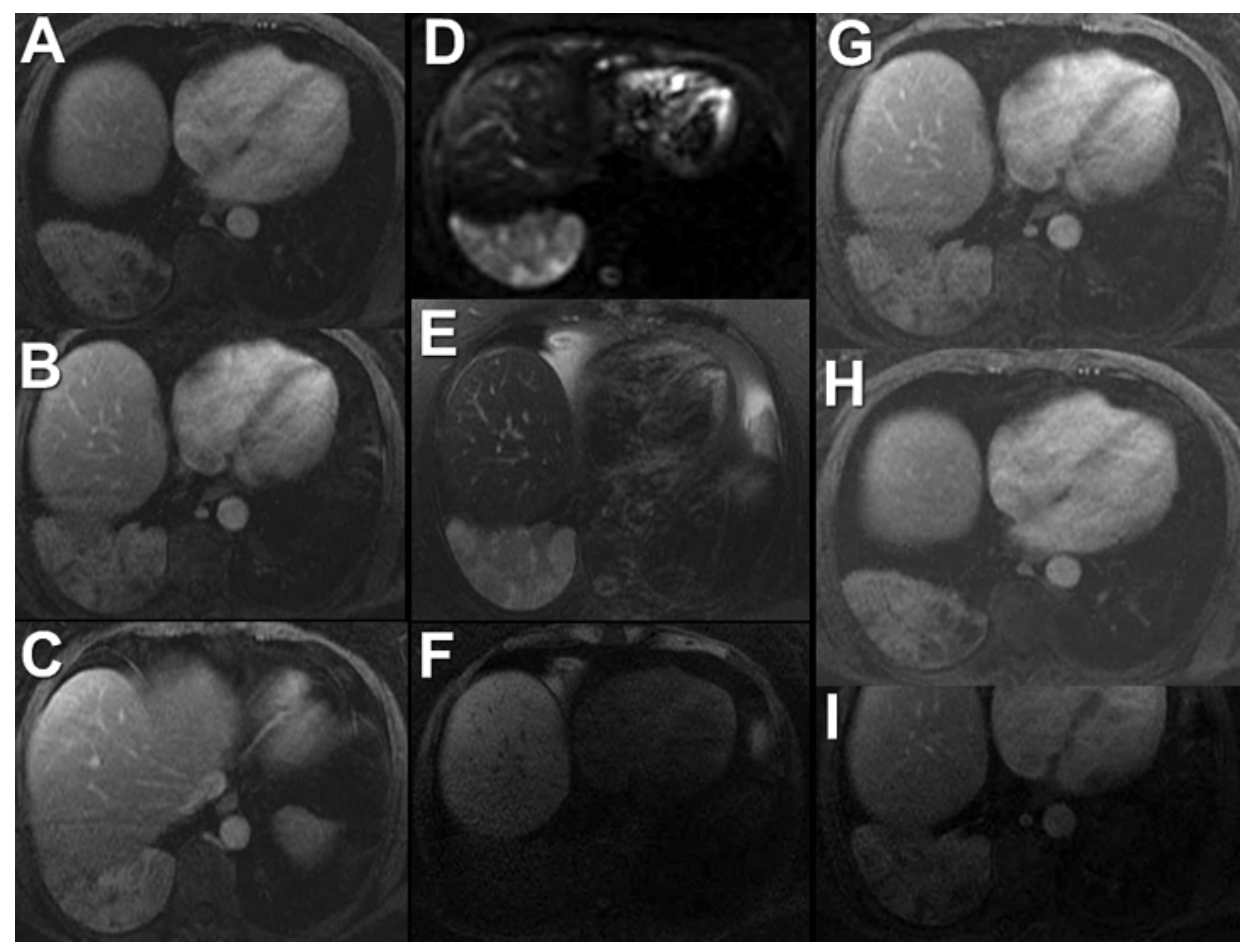

Figure 3. MR imaging using steady-state free precession imaging (A-C), diffusionweighted imaging (D), T2-weighted imaging with fat saturation (a fluid-sensitive sequence) (E), unenhanced (F) and enhanced (G-I) 3D fast spoiled gradient echo imaging shows the inhomogeneous mass in the right costophrenic angle. 
Which of the following regarding this MR examination is correct?

1. The abdominal MR shows additional sites of abnormality in the left thorax

2. The abdominal MR shows that the lesion does not enhance significantly

3. The abdominal MR shows that the lesion has locally aggressive behavior

4. The abdominal MR shows that the right-sided thoracic lesion is attached to a stalk

5. The abdominal MR shows the same mass at $\mathrm{CT}$, but adds little additional information to that already available with CT 


\section{Correct!}

\section{The abdominal MR shows the same mass at $\mathrm{CT}$, but adds little additional information to that already available with CT}

The abdominal MR examination shows the same mass that was seen at thoracic CT. The mass clearly enhances- compare the unenhanced fast spoiled-gradient echo image (Figure 3, panel F) to the enhanced fast spoiled-gradient echo images (Figure 3, panels G-I). There is relatively little additional information available at this MRI examination compared with that already known at thoracic $\mathrm{CT}$. The mass has a broad contact with the posterior chest wall- the mass is not pedunculated and no stalk is visible. The mass appears relatively indolent- no locally aggressive features, such as chest wall invasion, chest wall destruction, or significant mass effect- are evident. No other sites of abnormal signal are present on this MR examination.

Which of the following represents an appropriate next step for the evaluation of this patient?

1. ${ }^{68} \mathrm{Ga}$-citrate scanning

2. ${ }^{99 m}$ Tc-MAA ventilation-perfusion scintigraphy

3. Inspiratory and expiratory chest radiography

4. Lateral decubitus CT

5. Thoracic CT 


\section{Correct! \\ 5. Thoracic CT}

The right thoracic lesion was fairly well seen at the abdominal CT and MR examinations, but it would be useful to determine if other sites of disease are present to formulate differential considerations and develop an approach to establishing a diagnosis. Inspiratory and expiratory radiography is primarily used for detection of pneumothorax or to assess for air trapping, such as in the context of a suspected foreign body within the large airways, and would not play a role here. ${ }^{68} \mathrm{Ga}$-citrate scintigraphy is occasionally used for assessment of diffuse lung opacities, perhaps to detect opportunistic infections in immunocompromised patients, or pneumonitis related to medication-induced pulmonary injury, but neither are appropriate considerations for this patient. ${ }^{99 m}$ Tc-MAA ventilation-perfusion scintigraphy is useful for the detection of suspected thromboembolic disease but pulmonary embolism is not highly suspected in this patient as the morphology of the right thoracic lesion is not suggestive of an infarct; therefore ${ }^{99 m}$ Tc-MAA ventilation-perfusion scintigraphy is not the most appropriate next step. Lateral decubitus thoracic CT can be useful to assess for the mobility of pleural liquid or to assess for pneumothorax or air trapping, but these are not relevant diagnostic considerations for the mass in this patient.

The patient underwent contrast-enhanced thoracic CT (Figures 4 and 5).

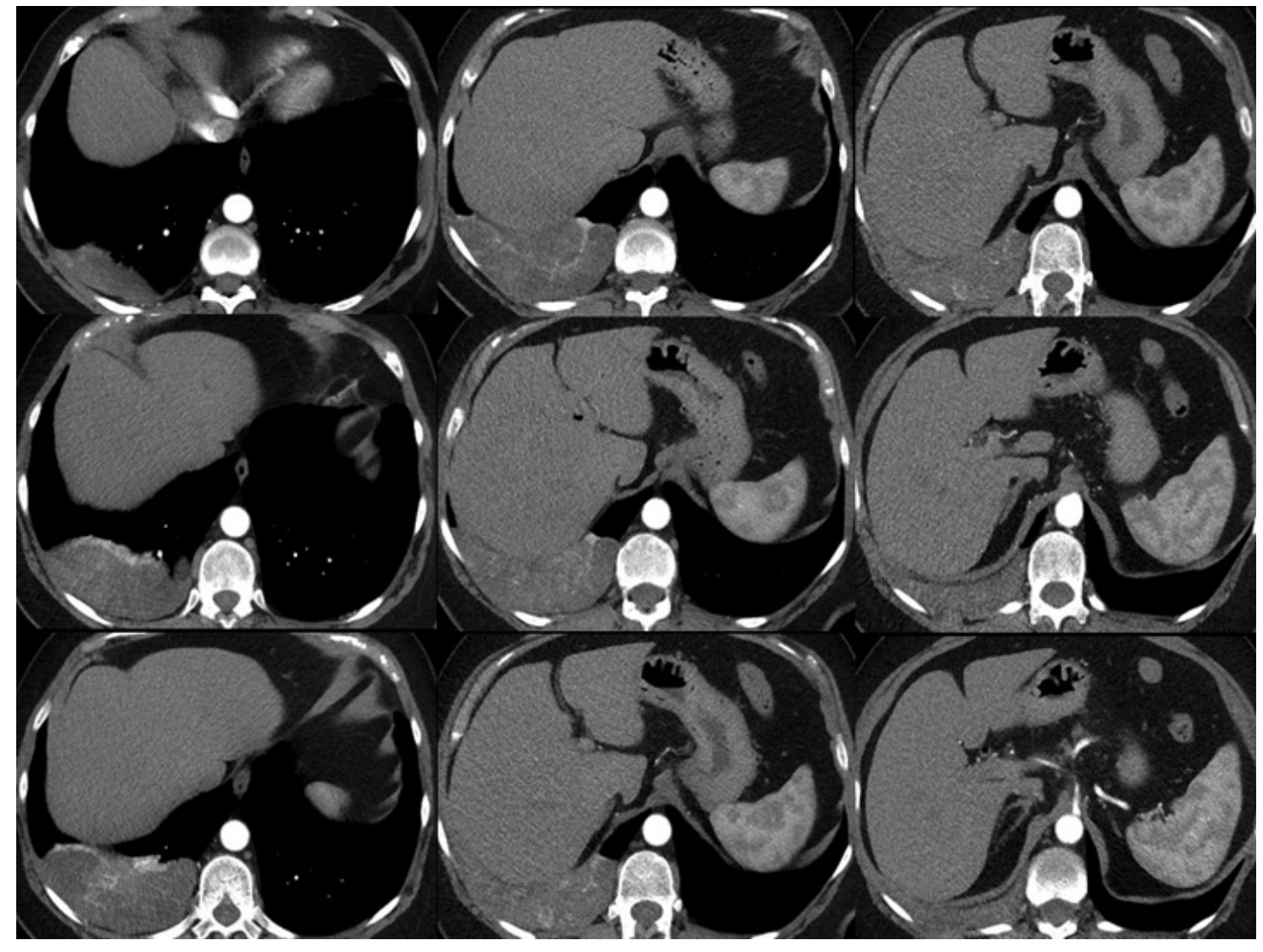

Figure 4. Axial enhanced thoracic CT. 


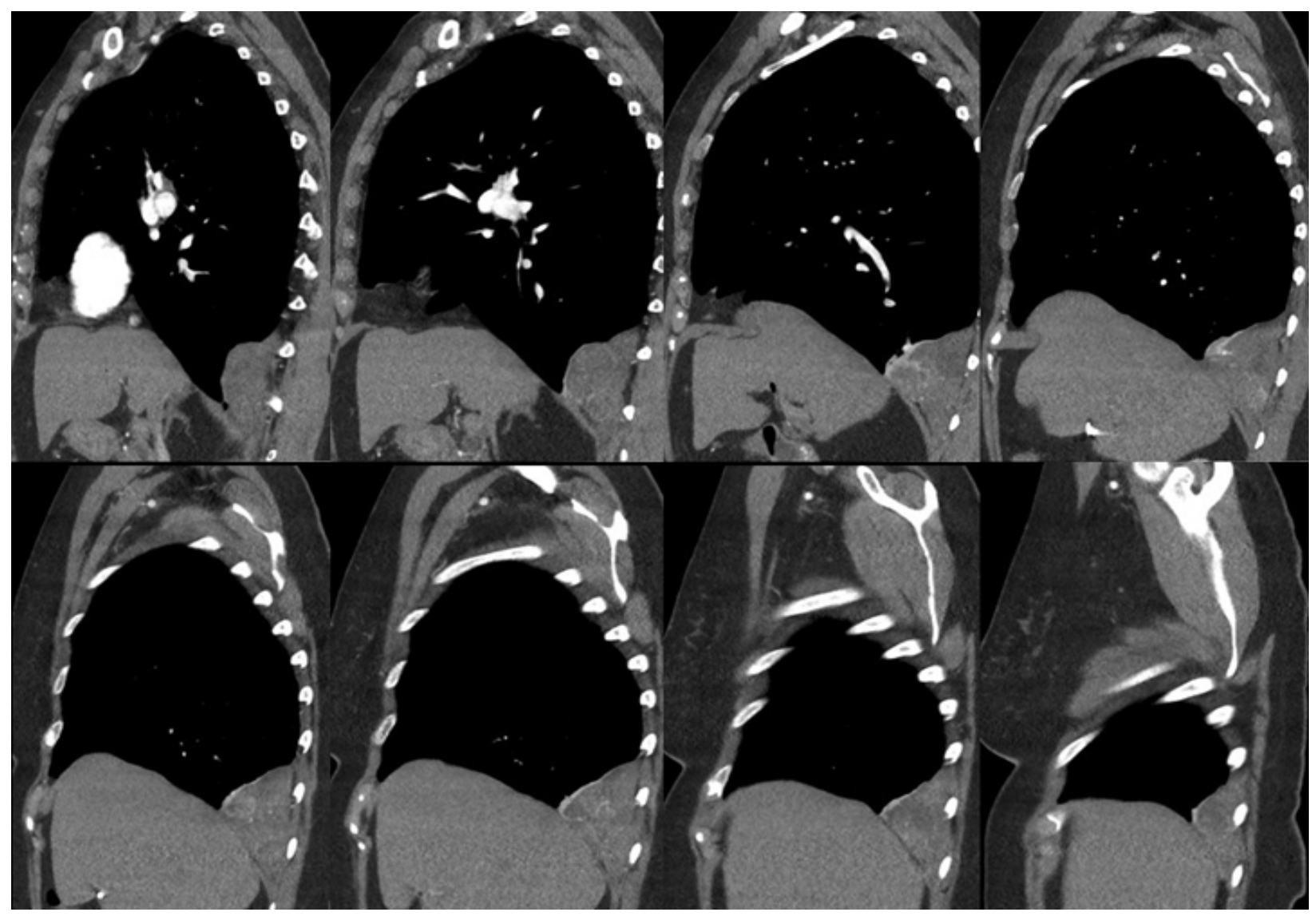

Figure 5: Sagittal enhanced thoracic CT.

Which of the following statements regarding the thoracic CT is most accurate?

1. The enhanced thoracic CT shows a heterogeneously enhancing right base thoracic lesion located within the posterior right costophrenic sulcus.

2. The enhanced thoracic CT shows additional sites of abnormality in the mediastinum

3. The enhanced thoracic CT shows an aberrant vessel arising from the abdominal aorta supplying the right thoracic lesion

4. The enhanced thoracic CT shows foci of gas within the right thoracic lesion

5. The enhanced thoracic CT shows the right thoracic lesion has changed shape and shifted location compared with the previous abdominal CT and MR examinations 


\section{Correct! \\ 1. The enhanced thoracic $C T$ shows a heterogeneously enhancing right base thoracic lesion located within the posterior right costophrenic sulcus.}

The enhanced thoracic CT shows the heterogeneously enhancing right base mass, again with a broad contact with the right posterior chest wall, located within the right posterior costophrenic sulcus, but without other visible abnormalities or locally aggressive behavior. The mass appears very similar to that seen at the prior CT and MR examinations- the lesion has not "changed shape" or shifted positions since those prior studies. The right thoracic lesion is vascularized, but no aberrant vessel supplying the mass, either from the descending thoracic aorta or abdominal aorta, is seen. The mass is heterogeneous, with areas of relatively lower attenuation mixed with areas of hyperattenuating enhancement, but no foci of fat or gas are present.

Which of the following represents the next most appropriate step for the evaluation of this patient?
1. ${ }^{18}$ FDG-PET
2. ${ }^{68} \mathrm{Ga}$-citrate scintigraphy
3. Right decubitus chest radiography
4. Thoracentesis
5. Video-assisted thoracoscopic surgery 


\section{Correct! \\ 1. ${ }^{18}$ FDG-PET}

Among the choices listed, ${ }^{18}$ FDG-PET would be the most useful procedure as it has the ability to assess for metabolic activity within the lesion as well as the ability to detect potential sites of disease elsewhere within and outside the thorax. Right lateral decubitus chest radiography is primarily useful for detection and quantification of right pleural fluid (the dependent side of the thorax in this example) or for detection of leftsided pneumothorax (the non-dependent thorax in this example); neither are considerations for this patient. ${ }^{68} \mathrm{Ga}$-citrate scintigraphy again would not provide addition useful information in this patient. Video-assisted thoracoscopic surgery would be a useful procedure for obtaining a diagnosis for this patient, but there may be less invasive approaches to obtaining a diagnosis for this patient. Because the lesion appears complex and solid, thoracentesis is not an appropriate procedure for evaluation of this lesion.

The patient subsequently underwent ${ }^{18}$ FDG-PET scanning (Figure 6).

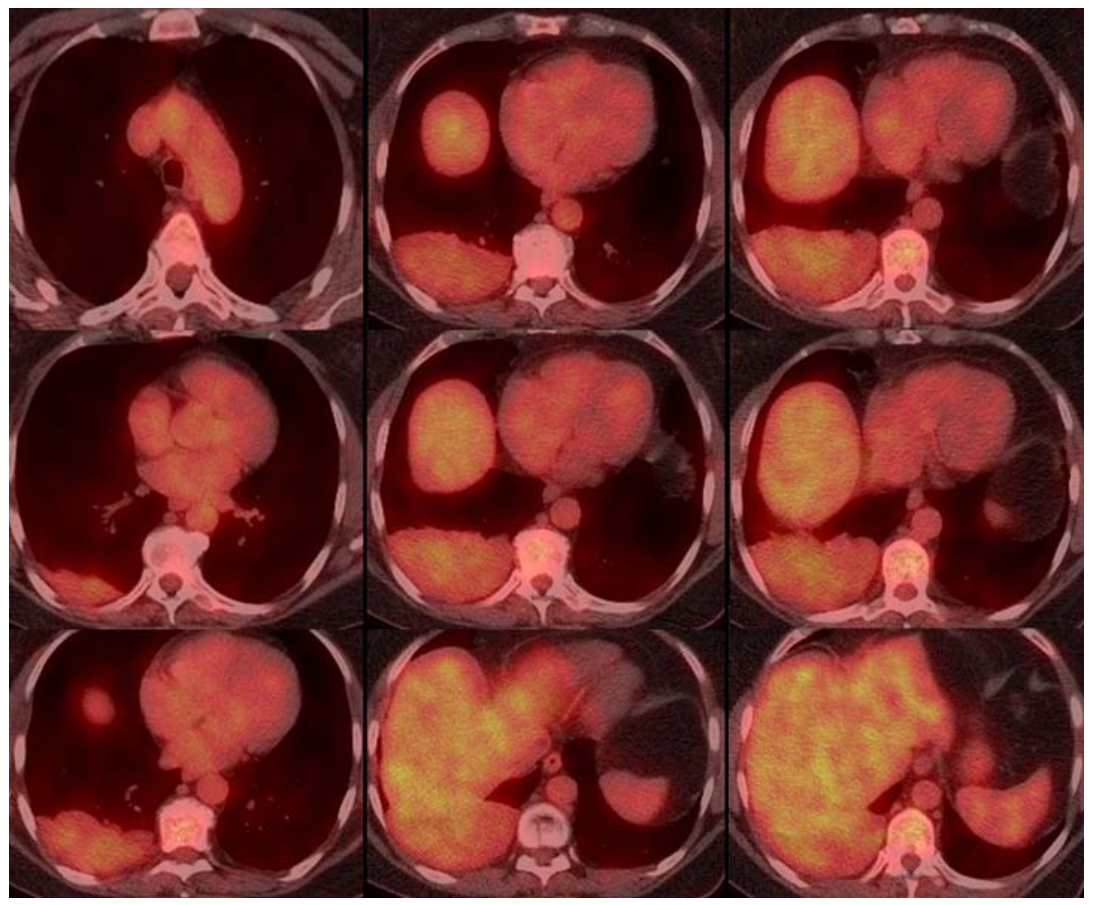

Figure 6. Axial ${ }^{18}$ FDG-PET.

Which of the following now represents the most likely diagnosis for this patient?

1. Bronchogenic malignancy

2. Malignant pleural mesothelioma

3. Metastatic disease

4. Neurogenic tumor

5. Solitary fibrous tumor of the pleura 


\section{Correct! \\ 5. Solitary fibrous tumor of the pleura}

The right thoracic lesion is a solitary, indolent-appearing mass with extensive pleural contact, the latter feature suggesting a pleural or chest wall origin, rather than a pulmonary origin, as would be the case for bronchogenic malignancy. The lesion shows no locally aggressive behavior. These features would not be expected for metastatic disease (and the patient has no evidence of primary malignancy outside the thorax), bronchogenic malignancy, or malignant pleural mesothelioma. The described features are, however, quite typical of solitary fibrous tumor of the pleura. Neurogenic lesions are typically located in the posterior mediastinum when arising within the thorax, and therefore would be expected to have a closer association with the spine. It is possible that a neurogenic tumor arising from the intercostal nerve could have an appearance similar to this lesion, although such neurogenic lesions are usually smaller and rounded or more elliptical / lenticular in appearance rather than the lobulated morphology displayed by the lesion in this patient. Further, the sagittal CT imaging shows the right thoracic lesion in this patient to conform very closely to the posterior pleural space, which favors a pleural origin for the lesion.).

Based on the data thus far, which of the following represents the next most appropriate step for the evaluation of this patient?

1. Endoscopic ultrasound

2. Flexible fiberoptic bronchoscopy

3. Mediastinoscopy

4. Percutaneous transthoracic fine needle aspiration and core biopsy

5. Video-assisted thoracoscopic surgery 


\section{Correct!}

\section{Percutaneous transthoracic fine needle aspiration and core biopsy}

Given the peripheral nature of this lesion and the lack of an associated airway, flexible fiberoptic bronchoscopy is not the best method for obtaining a tissue diagnosis for the lesion in this patient. Mediastinoscopy cannot access the inferior thorax caudal to the posterior aspect of the subcarinal space, and the lesion in this patient has no mediastinal extension. Similarly, the lesion does not have contact with, and is not in proximity to, the esophagus, and therefore endoscopic ultrasound would not be a good choice for obtaining a tissue diagnosis in this patient. Video-assisted thoracoscopic surgery would be a useful procedure for obtaining a tissue diagnosis for this patient, but a tissue diagnosis could also be achieved less invasively using percutaneous transthoracic fine needle aspiration and core biopsy; the latter is the best method among those listed for obtaining a diagnosis for this patient.

Further clinical course: The patient underwent percutaneous transthoracic fine needle aspiration and core biopsy which showed a small spindle cell neoplastic process associated with areas of collagen, but no evidence of necrosis. Immunoperoxidase staining showed positivity for CD34, BCL2, CD99 and rarely faintly positive estrogen receptors, and pancytokeratin staining was negative. The histopathologic features are consistent with solitary fibrous tumor of the pleura.

Diagnosis: Solitary fibrous tumor of the pleura.

\section{References}

1. Ginat DT, Bokhari A, Bhatt S, Dogra V. Imaging features of solitary fibrous tumors. AJR Am J Roentgenol. 2011; 196(3):487-95. [CrossRef] [PubMed]

2. Sung SH, Chang JW, Kim J, Lee KS, Han J, Park SI. Solitary fibrous tumors of the pleura: surgical outcome and clinical course. Ann Thorac Surg. 2005; 79(1):303-7. [CrossRef] [PubMed]

3. Truong M, Munden RF, Kemp BL. Localized fibrous tumor of the pleura. AJR Am J Roentgenol. 2000; 174(1):42. [CrossRef] [PubMed]

4. Chick JF, Chauhan NR, Madan R. Solitary fibrous tumors of the thorax: nomenclature, epidemiology, radiologic and pathologic findings, differential diagnoses, and management. AJR Am J Roentgenol. 2013; 200(3):W238-W248. [CrossRef] [PubMed]

5. Bhardwaj $\mathrm{H}$, Lindley S, Bhardwaj B, Carlile PV, Huard DR. Catch me if you can: a wandering solitary fibrous tumor of the pleura. Am J Respir Crit Care Med. 2014; 190(3):e7-9. [CrossRef] [PubMed]

6. Cardillo G, Lococo F, Carleo F, Martelli M. Solitary fibrous tumors of the pleura. Curr Opin Pulm Med. 2012; 18(4):339-46. [CrossRef] [PubMed] 